\title{
Fused primary first mandibular macromolar with a unique relation to its permanent successors: A rare tooth anomaly
}

\author{
Abhishek Dhindsa ${ }^{1}$, Shalini Garg ${ }^{1}$, S. G. Damle ${ }^{1}$, Shireen Opal ${ }^{1}$, Tavleen Singh ${ }^{1}$
}

Correspondence: Dr. Abhishek Dhindsa

Email: abhishekdhindsa@yahoo.co.in
'Department of Pediatric and Preventive Dentistry, Maharishi Markandeshwar College of Dental Sciences and Research, Mullana, Ambala, Haryana, India

\section{ABSTRACT}

Dental anomalies of number and forms may occur in the primary and permanent dentition. Various terms have been used to describe dental twinning anomalies: Germination, fusion, concrescence, double teeth, conjoined teeth, twinned teeth, geminifusion, and vicinifusion. Fused tooth is a developmental anomaly that is seen more frequently in the primary than the permanent dentition. Double tooth involving deciduous anterior teeth is found mostly in the mandible. Very few cases of nonsyndromic double primary molar have been reported in the literature. The succeeding permanent tooth is often found missing congenitally in the same region. This article reports a very rare unilateral occurrence of an anomalous, primary mandibular first macromolar formed by fusion with a dysmorphic premolar like supernumerary tooth in deciduous dentition period. Instead of agenesis of succedaneous tooth, the double tooth has been succeeded by normally developing mandibular first premolar in the same region.

Key words: Fusion, nonsyndromic, supernumerary

\section{INTRODUCTION}

The prevalence of double teeth in primary dentition is reported to be in the range of $0.1-3 \%$, with no sex predilection. ${ }^{[1-4]}$ Double teeth have been reported predominantly in the incisor and canine regions. They can be seen unilaterally or bilaterally in either the maxillary or mandibular dentition. Although esthetic and functional problems resulting from double primary teeth are transient, proper monitoring of dental development is necessary to prevent malocclusion resulting from the influence of large fused teeth on tooth alignment and arch symmetry, especially when supernumerary teeth are involved. ${ }^{[5-7]}$ Some studies have shown a proportion of permanent successor anomalies up to $50 \%$ following primary double teeth, including congenitally missing teeth, supernumerary teeth, and repeated double teeth formation. ${ }^{[8-10]}$ The nature of any such changes in the dentition as a result of various factors usually depends on the timing of insult in relation to the developmental stage of the affected teeth. Mandibular deciduous first molar is interesting in its resemblance to a transitional stage between primitive and present hominid molari form teeth. Initial calcification of mandibular deciduous molar starts at 15.5 weeks of intrauterine life and crown is completed by 5.5 months, while root completion occurs by 2.5 years. A rare anomaly, fusion of primary molar and supernumerary premolar, is observed in the present case, possibly formed by union of two discrete tooth buds to form a tooth with an anomalous shape with partially shared enamel and dentin.

\section{CASE REPORT}

A 5-year-old male child of Indo-Aryan origin reported to Pediatric Dentistry clinic, with the chief complaint of discomfort on chewing in the lower left back region since 3-4 months, with intermittent pain and discharge for last 4-5 days and multiple decayed teeth. Boggy swelling in

\footnotetext{
How to cite this article: Dhindsa A, Garg S, Damle SG, Opal S, Singh T. Fused primary first mandibular macromolar with a unique relation to its permanent successors: A rare tooth anomaly. Eur J Dent 2013;7:239-42.

Copyright $\odot 2013$ Dental Investigations Society. DOI: $10.4103 / 1305-7456.110195$
} 
buccal gingiva in relation to pulpally involved primary mandibular left first molar and primary mandibular right first molar due to caries was evident. An abnormal, unexplainable, painful hard swelling was palpable in the attached gingiva lingually in relation to this macrodont primary mandibular left first molar [Figure $1 \mathrm{a}$ and $\mathrm{b}$ ]. Patient's medical history was insignificant and no history of facial trauma was reported.

Clinical examination showed that the patient was suffering from rampant caries and all 20 primary teeth were present in the oral cavity. Full mouth rehabilitation was planned. Primary mandibular first molar of the left side was specifically examined for its anomalous, aberrant, and large clinical crown showing abnormal cuspal and fissure anatomy, compared to its contralateral tooth [Figure $1 \mathrm{a}$ and $\mathrm{b}$ ].

Radiographic examination of this fused primary mandibular left first molar revealed an enlarged primary molar crown with divided pulpal chambers by two individual crowns. Three distinct roots were noted in continuation with these two different crowns. Another normally developing and positioned permanent mandibular left first premolar was visible radiographically [Figure 2]. None of the permanent teeth were absent. The radiograph revealed supernumerary tooth fused lingually by its crown and root running between mesial and distal root of primary mandibular left first molar. It could be well appreciated that a fully formed supernumerary premolar of smaller size with single root was partially fused with primary mandibular left first molar.

In this case, the number of teeth in the dental arch was also normal and differentiation from gemination was clinically difficult or impossible. Primary mandibular left first molar was greatly predisposed to caries and periodontal disease in this child already suffering from severe rampant caries. Aberrant tooth anatomy of primary mandibular left first molar made endodontic treatment impossible. As patient presented with reoccurring buccal and lingual abscess in the attached gingiva below the fused tooth, nonsurgical extraction was done and space management was planned for the extracted space[Figure 3]. Primary first mandibular molar from mesial aspect showed characteristic feature of the extreme curvature buccally at cervical third [Figure 4a]. An extra prominent cusp resembling middle lobe of a mandibular premolar could be appreciated mesiolingually from this aspect. Both the mesiobuccal cusp of primary mandibular left first molar and distinct cuspal ridge of this rudimentary premolar supported by a root could also be visualized. It appeared that an extra rudimentary fully formed mandibular premolar was fused partially to mesiolingual aspect of primary mandibular left first molar [Figures $4 \mathrm{~b}$ and $4 \mathrm{~d}$ ]. Occlusal outline of this tooth showed abnormally

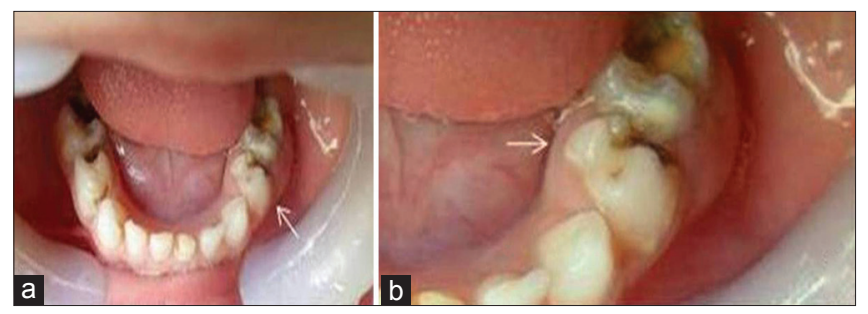

Figure 1: (a) Clinical picture of the mandibular arch showing unilateral presence of fused macromolar L. Difference in shape can be appreciated by comparing with $S$, (b) Clinical crown shows extra mesio-lingual cusps of $\mathrm{L}$ resembling a rudimentary premolar with abnormal bulge and swelling in relation its attached gingival lingually.

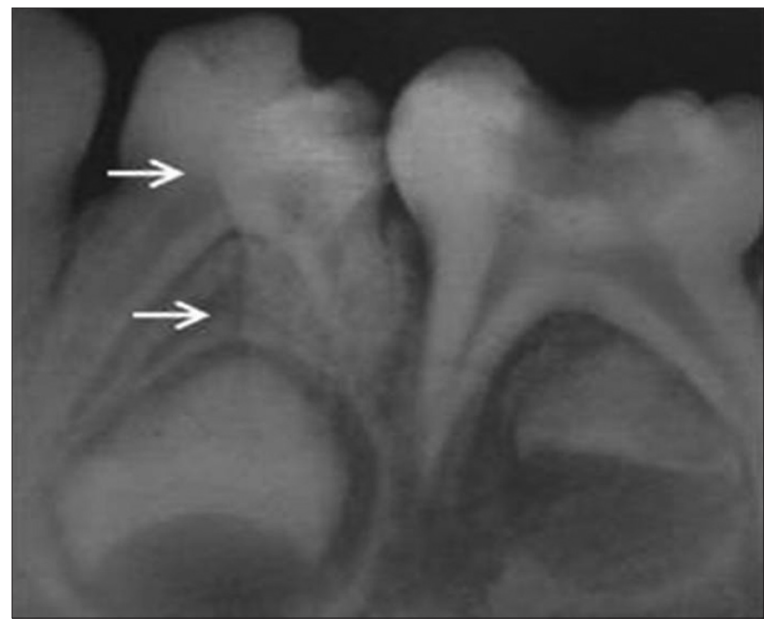

Figure 2: IOPA radiograph showing fused macromolar L, which is partially fused to a supernumerary tooth resembling a bicuspid, with divided pulp chamber and 3 roots. Normally developing 21 can be well appreciated.

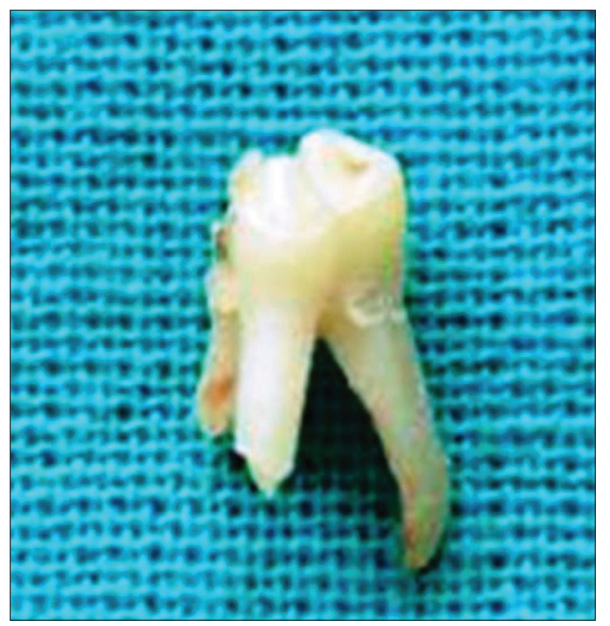

Figure 3: Anatomical view of extracted macrodont L, partially fused with an extra rudimentary premolar like tooth with prominent middle lobe, on the lingual aspect of its crown. 
large lingual side contributed by supernumerary fused tooth as compared to its contralateral tooth [Figuer 5]. An extra deep groove was separating cusp of supernumerary tooth and mesiolingual cusp of primary mandibular left first molar [Figures $1 \mathrm{~b}$ and $4 \mathrm{~d}$ ]. Distobuccal and distolingual cusps of this fused molar were destroyed by caries and pulpally involved [Figure $4 \mathrm{~b}$ ].

\section{DISCUSSION}

This case report presents a rare case of partial fusion of primary mandibular left first molar to supernumerary premolar of smaller size with normal presence of permanent mandibular left first premolar. No clear etiology is evident in the literature. In general, internal factors such as trauma, radiation, hormonal changes, and nutritional deficiencies could affect the shape, number, and quality of dentition. ${ }^{[11,12]}$ Genetic studies state that the specification of dental patterning is apparent in early mandibular epithelium. BMP4, expressed in epithelium overlying incisor fields, induces MSX-1, while FGF8, expressed over molar fields, induces Barx-1. It suggests that morphogenic fields play a role in determining the expression of canine and premolar teeth. These experiments suggest that there is importance of early epithelial signals in the specification of tooth type, while tooth shape is not predetermined prior to neural crest migration. ${ }^{[13]}$ Local factors during tooth bud development result in persistence of dental lamina. The etiology of the

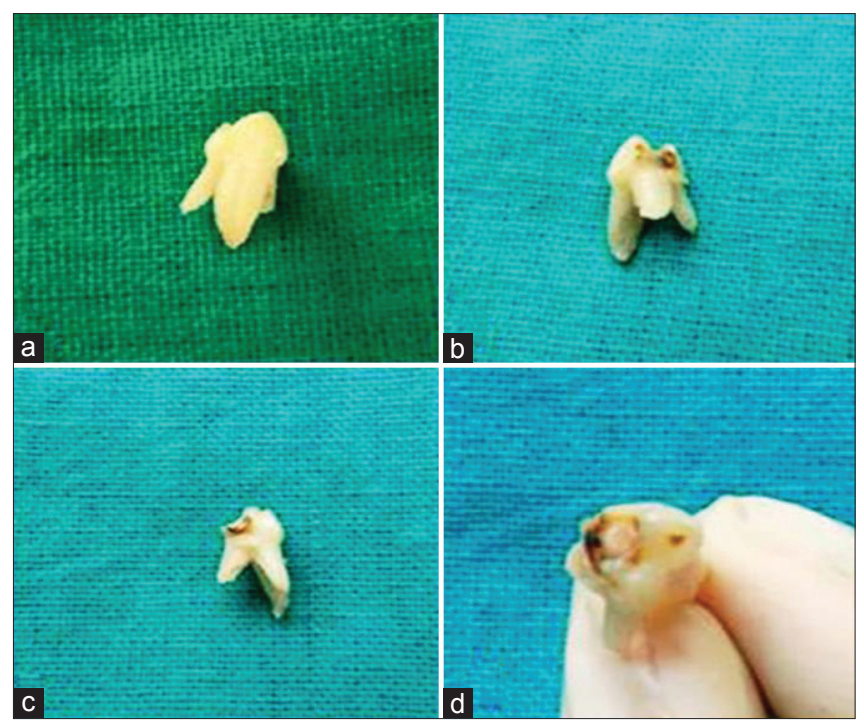

Figure 4: Various views of extracted macrodont L (a) Mesial aspect showing crown ridge and part of root of supernumerary rudimentary premolar (b) Lingual aspects showing deep carious lesion (c) Distolingual aspect (d) Occlusal aspect showing full anatomy of partially fused supernumerary rudimentary premolar. present case supports the view of fusion occurring somewhere very initially in utero. As crown part was partially fused with premolar like supernumerary tooth, it showed that this extra tooth was a result of hyperdontia believed to be associated with excess of dental lamina. Therefore, this report presents a rare case of primary molar fusion. The nature of the tooth morphology, the number of teeth present in the arch, and the radiographic findings all seem to coincide with previous definitions of fusion as proposed by Levitas ${ }^{[14]}$ and Mader. ${ }^{[15]}$ Three cases of molar fusion have been reported in the literature. The first case was reported by Yuen, et al..$^{[9]}$ in a retrospective radiographic study of 376 patients. The case reported by Acs, et al. ${ }^{[16]}$ showed clear buccal and lingual grooving of the crown, indicating where the primary molars were joined. The occlusal morphology of this case also revealed the anatomies of the first and second molars. Radiographically, this case exhibited two seemingly distinct pulp chambers, incomplete fusion of the dentin, and possibly five or six roots of the macrodont. Another reported case of macrodont of primary maxillary second molar demonstrated complete fusion of primary molars. This macrodont included a total of six cusps, three buccal and three lingual, which were separated by occlusogingival grooves with a single pulpal chamber and four distinct roots. ${ }^{[17]}$ The literature indicates a tendency for missing permanent successors in cases of primary tooth fusion rather than an extra tooth. The present case is the rarest of rare cases as it exhibits unilateral presence of macrodont primary mandibular left first molar formed by clinically evident partial fusion with supernumerary premolar of smaller size showing two seemingly distinct pulp chambers, incomplete

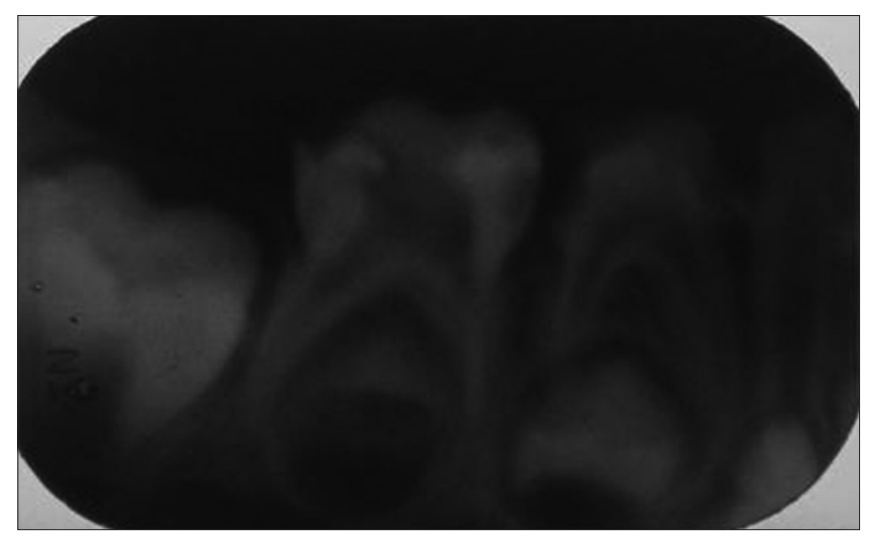

Figure 5: The radiographic image of contralateral right mandibular molar is provided. It has only two roots with nor mal crown dimension which can also be seen in the clinical photograph. The dimension, presence of three roots, site of attachment of premolar and all side image of extracted tooth confirmed the fusion of mandibular first molar with supernumerary tooth. 
fusion of the dentin and enamel, three distinct roots, and presence of normally developing permanent successor on radiographic examination. Aberrant tooth anatomy of primary mandibular left first molar made endodontic treatment impossible. As the patient presented with recurring buccal and lingual abscess in attached gingiva below the fused tooth, nonsurgical extraction was done and space management was planned for the extracted space.

\section{CONCLUSION}

This paper highlights the following points:

- An unusual location for dental fusion (unilateral, mandibular primary first molar region)

- A type of variation seen in molar fusion (partial by crown as compared to complete)

- An unusual finding in primary tooth fusion (primary tooth and an apparent supernumerary eumorphic bicuspid)

- Normal presence of underlying successor

\section{REFERENCES}

1. Duncan WK, Helpin ML. Bilateral fusion and gemination: A literature analysis and case report. Oral Surg Oral Med Oral Pathol 1987;64:82-7.

2. Tasa GL, Lukacs JR. The prevalence and expression of primary double teeth in western India. ASDC J Dent Child 2001;68:196-200.

3. Hagman FT. Anomalies of form and number, fused primary teeth, a correlation of the dentitions. ASDC J Dent Child 1988;55:59-61.

4. Buenviaje TM, Rapp R. Dental anomalies in children: A clinical and radiographic survey. ASDC J Dent Child 1984;51:42-5.

5. Mattos-Garner RO, Rontani RM, Gavião MB, de Souza Filho FJ, Granatto AP, de Almeida OP. Anomalies of tooth form and number in the permanent dentition: Report of two cases. ASDC J Dent Child 1997;64:298-302.

6. Gazit E, Lieberman MA. Macrodontia of maxillary central incisors: Case reports. Quintessence Int 1991;22:883-7.

7. Sivolella S, Bressan E, Mirabal V, Stellini E, Berengo M. Extraoral endodontic treatment, odontotomy and intentional replantation of a double maxillary lateral permanent incisor: Case report and 6-year follow-up. Int Endod J 2008;41:538-46.

8. Nik-Hussein NN, Abdul Majid Z. Dental anomalies in the primary dentition: Distribution and correlation with the permanent dentition. J Clin Pediatr Dent 1996;21:15-9.

9. Yeun SWH, Chan JCY, Wei SH. Double primary teeth and their relationship with the permanent successors: A radiographic study of 376 cases. Pediatr Dent 1987;9:42-52.

10. Gellin ME. The distribution of anomalies of primary anterior teeth and their effect on the permanent successors. Dent Clin North Am 1984;28:69-80.

11. Navia JM. Research advances and needs in nutrition in oral health and disease. In Nutrition in Oral Health and Disease. In: Pollack RL Kravitz E, editors. Philadelphia: Lea and Febiger; 1985. P. 426-67.

12. Parkin SF. Traumatic injuries to the teeth. In Notes on Paediatric Dentistry. Oxford: Wright; 1991. p. 140-1.

13. Avery JK. Oral Development and Histology. $3^{\text {rd }}$ ed. Thieme Medical Publishers; 2002.

14. Levitas TC. Gemination, fusion, twinning and concrescence. J Dent Child 1965;32:93-100.

15. Mader CL. Fusion of teeth. J Am Dent Assoc 1979;98:62-4.

16. Acs G, Cozzi E, Pokala P. Bilateral double primary molars: Case report. Pediatr Dent 1992;14:115-6

17. Caceda J, Creath C, Thomas J, Thornton J. Unilateral fusion of primary molars with the presence of a succedaneous supernumerary tooth: Case report. Pediatr Dent 1994;16:53-5.

\begin{tabular}{|l|l|}
\hline \multicolumn{2}{|c|}{ Access this article online } \\
\hline Quick Response Code: & Website: \\
\hline & www.eurjdent.com \\
\hline
\end{tabular}

